\title{
Rolling-Texture Development in Aluminium Single Crystals with Deformation-Banding Tendency
}

\author{
By Keisuke Ikeda* and Eihachiro Tanaka*
}

\begin{abstract}
Orientation changes of aluminium single crystals with a deformation-banding tendency were studied to get information about the influence of band formation on the rolling-texture development in fcc metals. Both (111)[110] and (112) [110] crystals split into two components which rotate in opposite directions about the [111] axis near the transverse direction, and they develop pairs of orientations $\{123\}<745\rangle$ and $\{011\}<112\rangle$, respectively. The (111) $[110] \sim 15^{\circ}$ crystal in which the rolling direction deviates $15^{\circ}$ from [110] also rotates about the same axis as the formers. The end texture is, however, composed of two irrational components. Besides, pole intensity for one component is higher than the other. The dominant component is in the range of orientational spread for polycrystalline aluminium, but the poles of the minor component deviate from the intensity maxima. On the other hand, it is confirmed that the (011)[111] crystal tends to rotate in both directions about the [111] axis parallel to the rolling direction. The orientions within the range between $\{111\}<110\rangle$ and $\{112\}<110\rangle$ would rotate about the [1]1] axis near the transverse direction as in the case of (111) [110], resulting in the formation of a limited fiber texture ranging from $\{123\}<745\rangle$ to $\{011\}<112\rangle$. Band formation in the orientations near $\{011\}<111>$ would build up a limited fiber texture ranging from $\{112\}<111>$ to $\{123\}<745>$. Both the fiber textures characterize the copper-type rolling texture. The analysis of slip rotations in which the compatibility of strains is taken into consideration also indicates the development of the copper-type rolling texture. Therefore, it is concluded that all $f c c$ metals tend to form the copper-type rolling texture.
\end{abstract}

(Received September 27, 1971)

\section{Introduction}

The deformation textures developed in single crystals have extensively been studied with $f c \varepsilon$ and $b c c$ metals. Among the $f c c$ metals the rolling textures of copper and aluminium single crystals were studied by a number of workers ${ }^{(1)(2)}$. However, most of studies were concerned only with stable orientations for a specific mode of deformation and particular initial orientations.

Barrett $^{(3) \sim(7)}$ found that the structure of cold-worked cubic metals had a banded appearance. The banded structures, which are named deformation bands, appear in microscopic dimension in polycrystalline metals, and in coarser form in single crystals. In spite of Barrett's suggestion ${ }^{(8)}$ on the significance of band formation for the texture development, it received little attention ${ }^{(9)}$ untill recently. In nearly all of the theories about the formation of deformation textures, the splitting of individual crystal into bands has been ignored. Recently, McHargue and Reed ${ }^{(10)}$ and Ahlborn ${ }^{(11)(12)}$ pointed out that the formation of deformation bands in crystals had an important influence on the development of the $\langle 111\rangle$ fiber texture in wires and rods of $f c c$ metals.

In the axially-symmetric deformation of single crystals by drawing or extrusion, deformation bands are formed for every orientation ${ }^{(13)}$. It is considered that

* Research Institute for Iron, Steel and Other Metals, Tohoku University, Sendai, Japan.

(1) H.Hu; R.S.Cline and S.R.Goodman : Recrystallization, Growth and Textures, ASM, Metals Park, Ohio, (1965), p. 295.

(2) I. L. Dillamore and W.T. Roberts : Met. Rev., 10(1965), 271.

(3) C.S. Barrett : Trans. AIME, 135 (1939), 296.

(4) C.S. Barrett and L.H. Levenson: Trans. AIME, 135 (1939), 327.

(5) C.S. Barrett and L. H. Levenson : Trans. AIME, 137 (1940), 112.

(6) C.S. Barrett : Trans. AIME, 137 (1940), 128.

(7) C.S. Barrett and F. W.Steadman : Trans. AIME, 147 (1942), 57.

Trans. JI M slip on different systems caused by the constrained plastic flow results in band formation ${ }^{(10)}$. On the other hand, it was found that, under plane strain deformation, the orientations lacking in slip systems which satisfy the compatibility of strain were favourable for the formation of deformation bands ${ }^{(14)}$. But it is not known whether a separate band formed under plane strain deformation behaves like an isolated crystal grain or not. In the present study, rotations of aluminium single crystals with deformation-banding tendency were examined to get information about the influence of band formation on the rolling-texture development in $f c c$ metals.

\section{Experimental Procedure}

In a previous paper ${ }^{(14)}$, the orientations which tend to form deformation bands under plane strain deformation were determined theoretically and they were experimentally confirmed to be valid. Five orientations were chosen out of these orientations for the present study. They are shown in the standard (001) stereographic projection of Fig. 1. Aluminium single crystal plates, $3 \mathrm{~mm}$ thick, were grown by the Bridgeman method. After the crystal orientations were checked by the back-reflection Laue method, they were sectioned

(8) C.S. Barrett : Structure of Metals, McGraw-Hill, New York, (1952), p. 465.

(9) G. Wassermann und J.Grewen : Texturen metallischer Werkstoffe, Springer-Verlag, Berlin, (1962), p. 430.

(10) R. E. Reed and C. J. McHargue : Trans. Met. Soc. AIME, 239 (1967), 1604 .

(11) H. Ahlborn : Z. Metallk., 56 (1965), 205.

(12) H. Ahlborn : Z. Metallk., 56 (1965), 411.

(13) K. Yagi, K. Tkeda and E. Tanaka : J. Japan Inst. Metals, 35 (1971), 1177 (in Japanese).

(14) M. Sugiyama and K. Ikeda : J. Japan Inst. Metals, 34 (1970), 786 (in Japanese).

1972 Vol. 13 


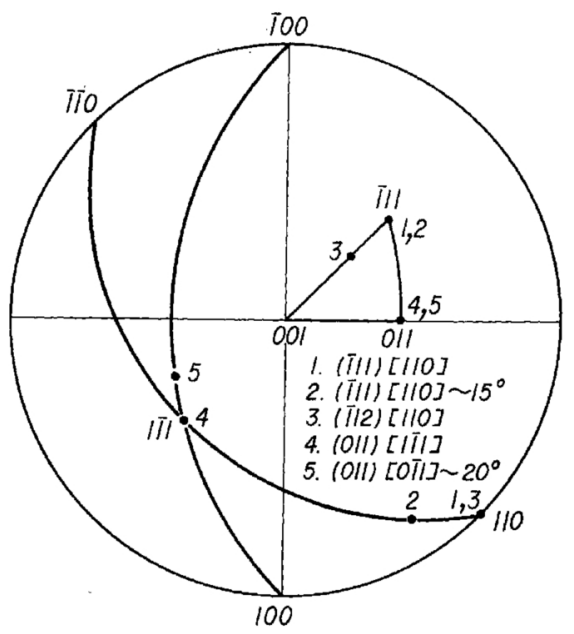

Fig. 1 Initial orientations of aluminium single crystals used for rolling texture studies.

into: $30 \mathrm{~mm}$ in width by $50 \mathrm{~mm}$ in length. All the specimens were electro-polished to remove the surface oxide layer. Then, each specimen was set in a polycrystalline aluminium flame to maintain plane strain deformation and was rolled by a constant reduction in thickness. Palm oil was used as the lubricant. At various reductions in thickness, specimens for texture determination were cut from the strips. The final reduction was 95 pct. Rolling was carried out at room temperature.

The specimens for texture determination were prepared by chemical-polishing and electro-polishing. $\{111\}$ pole figures were determined by the DeckerAsp-Harker method using $\mathrm{Cu}-\mathrm{K}_{\alpha}$ radiation. Intensity measurements were confined within $57^{\circ}$ from the circumference of the pole figure.

\section{Experimental Results}

\section{1. $(\overline{\mathbf{1} 11})[110\rfloor$ and $(\overline{\mathbf{1} 12})\lfloor 110\rfloor$ crystals}

The octahedral pole figure for the (I11) [110] aluminium single crystal rolled by 30 pct reduction in thickness is shown in Fig. 2. $\{111\}$ poles of the initial orientation are indicated by solid squares. For this crystal, a considerable spread in orientation is such that the lattice rotations about the [III] axis at $\sim 20^{\circ}$ from the transverse direction occur in both clockwise and counterclockwise directions. Correspondingly, its microstructure was characterized by many complex deformation bands. With increasing reduction, the crystal was completely split into two components, and they continued to rotate in opposite directions about the $[\bar{I} 1 \overline{1}]$ axis toward a pair of $\{123\}<745>$ orientations. Figure 3 represents the texture of the $(\overline{1} 11)[110]$ crystal rolled 60 pct. This crystal develops (I32)[754] and $(\overline{3} 12)[574]$ duplex orientations. This result is essentially consistent with Wonsiewicz-Chin's observation $^{(15)}$ on the orientational changes in the (111) [110] copper single crystal under plane strain compression. The $\{123\}<745>$ orientation remained stable to quite high reductions. It has been ascertained by selected-

(15) B. C. Wonsiewicz and G. Y. Chin : Met. Trans., 1 (1970), 2715.

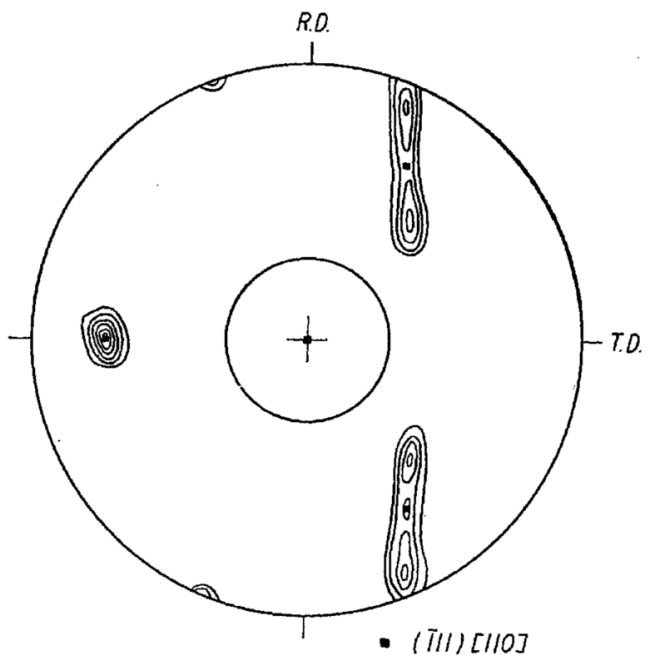

Fig. 2 Octahedral pole figure for the (111) [110] crystal rolled by 30 pct reduction in thickness. Solid squares indicate $\{111\}$ poles of the initial orientation.

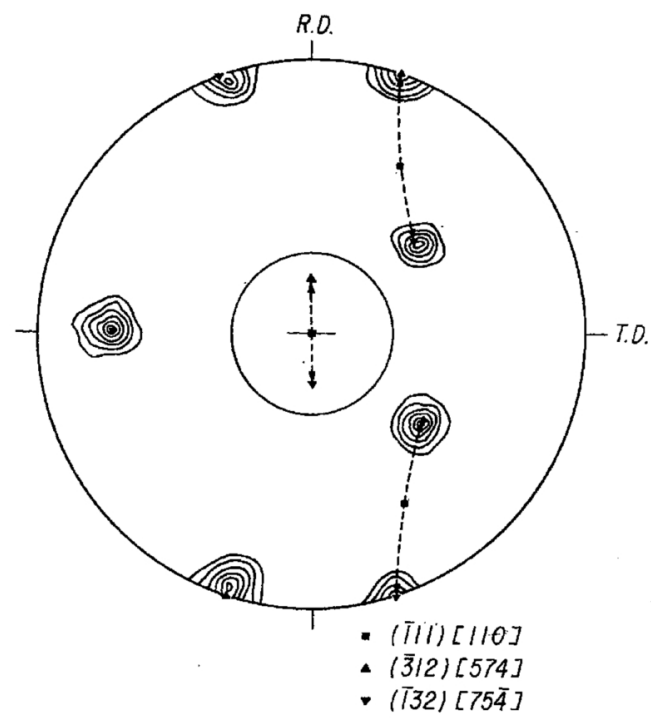

Fig. 3 Texture of the (111)[110] crystal rolled 60 pct. A pair of $\{123\}<745>$ orientations is developed.

area diffraction in the electron microscope $\mathrm{e}^{(16)(17)}$ that the $\{123\}<745>$ orientation is one of the dominant end orientations for $f c c$ metals of high stacking fault energy.

The single crystal with $(\overline{1} 12)[110]$ orientation also rotated in both directions about the same [I1 $\overline{1}]$ axis as in the case of the (I11)[110] crystal. After 60 pct reduction, two separate components attained to (011) [21I] and $(\overline{1} 01)[121]$ duplex orientations. This is shown in Fig. 4. Upon further rolling up to $95 \mathrm{pct}$ reduction, a pair of complementary $\{011\}<112>$ orientations remained with remarkable sharpness.

Since the orientations in the range of $\{112\}<110>$ to $\{111\}<110>$ have a tendency to form deformation bands, it can be predicted that they are characterized by lattice rotations in both directions about the $<111\rangle$ axis near the transverse direction.

(16) F. Haessner, U. Jakubowski und M. Wilkens : phys. stat. sol., 7 (1964), 701.

(17) K. Lücke, H. Perlwitz und W. Pilsche : phys. stat. sol., 7 (1964), 733. 


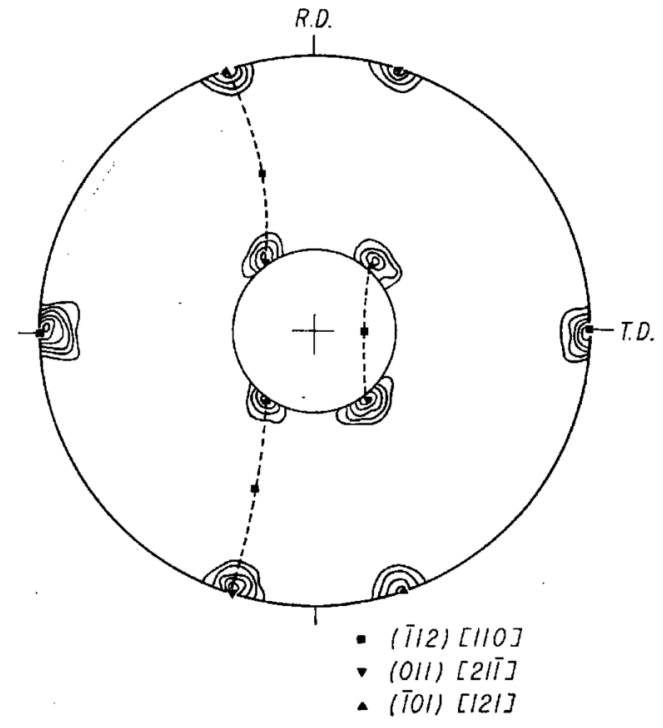

Fig. 4 Texture development in the (112)[110] crystal rolled 60 pct. Two separate components attain a duplex $\{011\}<112>$ end orientations.

\section{2. $(\overline{111})[110] \sim 15^{\circ}$ erystal}

It was shown in the previous study that crystals with their $\{111\}$ planes parallel to the rolling plane and their $<110\rangle$ axes lying within a range of $18^{\circ}$ from the rolling direction tended to form deformation bands. The $(\overline{1} 11)[110] \sim 15^{\circ}$ orientation is representative one of them. The pole figure in Fig. 5 shows the rolling texture of this crystal rolled 30 pet. The $(\overline{1} 11)[110] \sim$ $15^{\circ}$ crystal also indicates lattice rotations in both directions about the same axis as the formers, and it is completely divided into two components with increasing reduction. Microstructure also indicated complicatedly subdivided bands. With increasing reduction, the $[\overline{1} 1 \overline{1}]$ rotation axis shifted inward by a few degree on the octahedral pole figure. After 60 pct reduction, the rolling texture was composed of two irrational orientations as shown in Fig. 6 , and remained unchanged even after 95 pct reduction.

For both the $(\overline{1} 11)[110]$ and $(\overline{1} 12)[110]$ orientations, two complementary components gave the same intensity

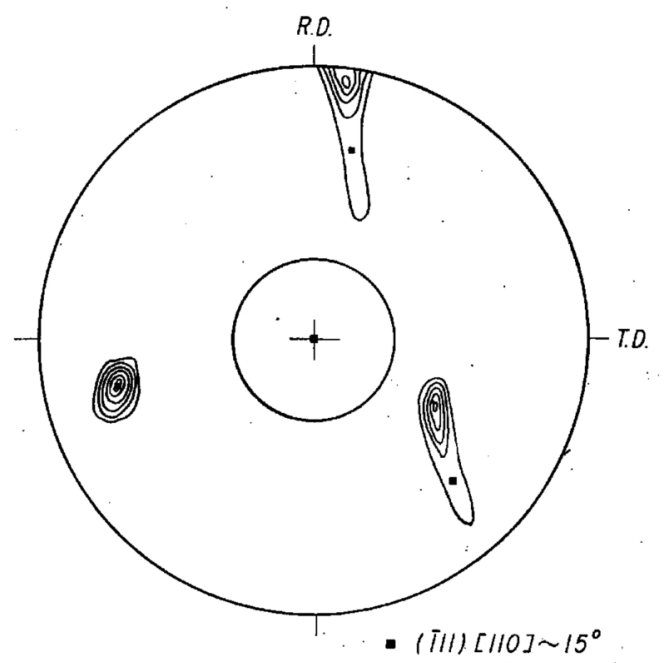

Fig. $5 \cdot\{111\}$ pole figure for the (111) $[110] \sim 15^{\circ}$ crystal rolled 30 pct.

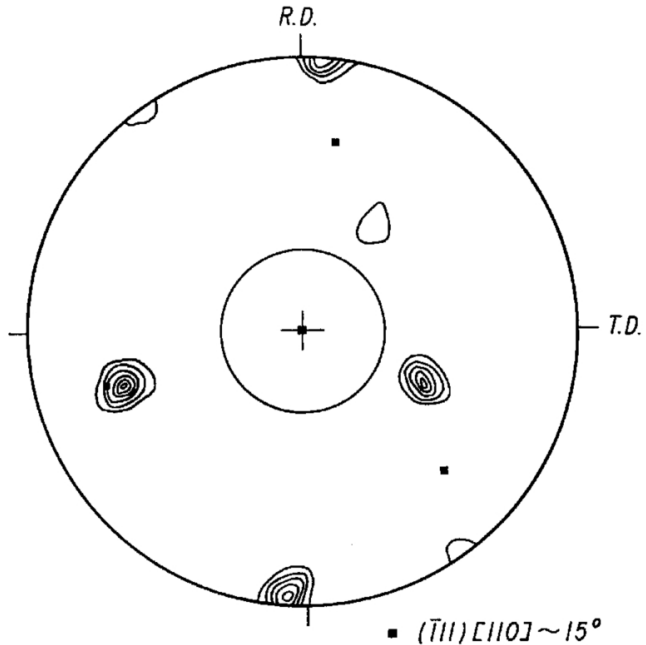

Fig. 6 Texture development in the $(111)[110] \sim 15^{\circ}$ crystal rolled 60 pct. Its texture is described by two irrational components.

on the pole figure. On the contrary, the rolling texture of the $(\overline{1} 11)[110] \sim 15^{\circ}$ crystal was composed of one dominant orientation and another minor orientation. $\{111\}$ poles of the dominant component are in the range of orientational spread on the pole figure for polycrystalline aluminium. On the other hand, those of the minor component deviate from the intensity maxima. The difference in the relative amount of two components is probably due to an unsymmetrical orientation the slip systems with respect to the stress axes. Both the orientations would be unstable if the fragmented bands would behave like individual grains during deformation. It is very interesting to note that two irrational orientations resulting from the band formation remain stable up to quite high reductions. It appears that there are a number of other orientations which behave in the same way as the $(\overline{1} 11)[110] \sim 15^{\circ}$ orientation. In a polycrystalline aggregate, it is quite likely that the grains with such orientations contribute to the scatters of preferred orientations or the back-ground intensity. The above-mentioned evidence suggests that the band formation complicates the development of rolling textures.

\section{3. $(011)[1 \overline{1} 1]$ and $(011)[1 \overline{2} 2]$ crystals}

Figure 7 shows the octahedral pole figure for a (011) [1II] crystal rolled by 30 pct reduction in thickness. A considerable orientational spread is observed, but this crystal indicates the trend of lattice rotation toward (011)[1] 2$]$ about the sheet normal. After 60 pet reduction, orientation spreads in both directions occur as shown in Fig. 8.

However, experiments performed on several specimens of the same orientation showed that the smaller the lattice rotation about the sheet normal, the more striking the lattice rotation about the [1 11$]$ axis near the rolling direction. This result suggests that even the (011) [1]1] crystal forms deformation bands and the bands rotate in opposite directions about the (1III) axis. The rotation about the sheet normal at small reductions might be caused by non-plane strain deformation because of 


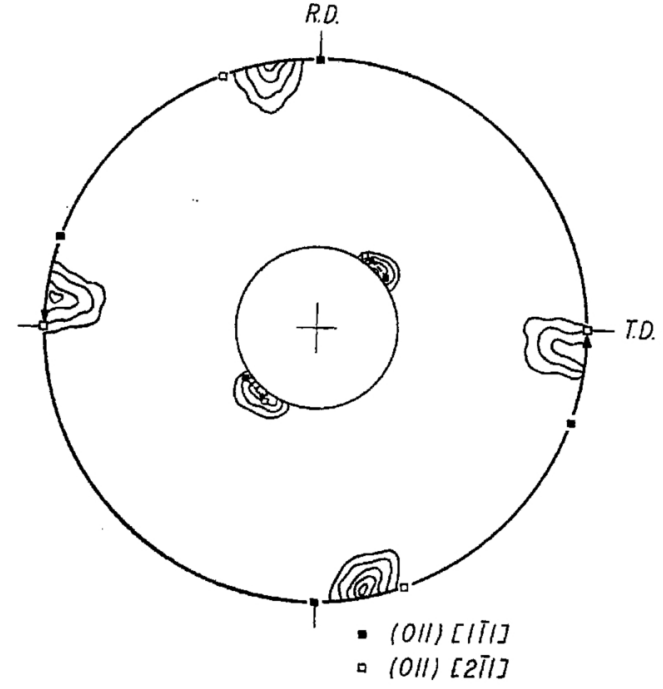

Fig. $7\{111\}$ pole figure for the (011) [1111] crystal rolled $30 \mathrm{pct}$. It tends to rotate about the sheet normal.

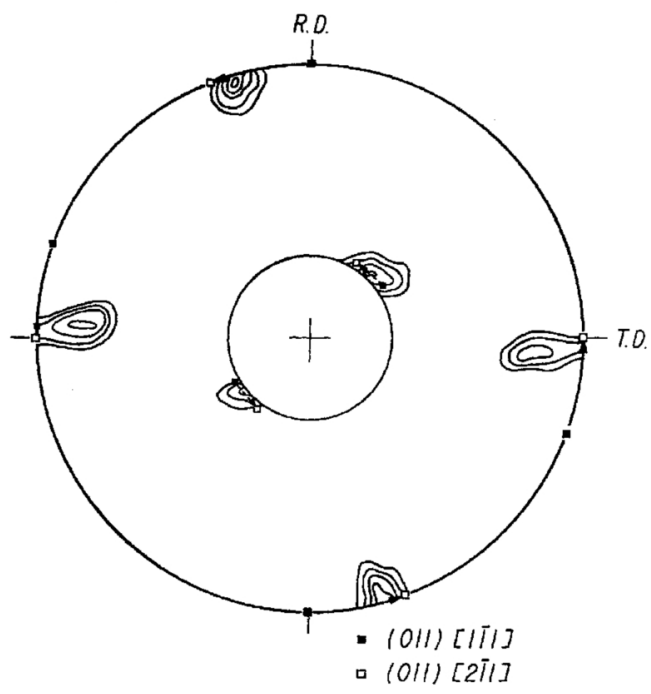

Fig. $8\{111\}$ pole figure for the $(011)[1 \overline{1} 1]$ crystal rolled 60 pct. It shows the orientational spread about the [1,111] axis.

the incomplete constraint. To confirm it, an experiment in the $(011)[0 \overline{1} 1] \sim 20^{\circ}$ orientation was carried out. The rolling direction which deviates $20^{\circ}$ from $[0 \overline{1} 1]$ corresponds to $[1 \overline{2} 2]$. The $(011)[0 \bar{I} 1] \sim 20^{\circ}$ orientation has no banding tendency and rotates simply toward (011)[1iㅣ] about the sheet normal during rolling with or without complete constraint. Therefore, it is expected that the crystal with this orientation is deformed under a plane strain condition before it attains the $(011)[1 \overline{1} 1]$ orientation with a banding tendency.

After 30 pct reduction, the $(011)[1 \overline{2} 2]$ crystal attained $(011)[1 \overline{1} 1]$ and indicated a spread about the [111] axis parallel to the rolling direction. With increasing reduction, the splitting of the intensity peak occurred, and the separated components continued to rotate in opposite directions about the $[1 \overline{1} 1]$ axis. After 70 pct reduction, they attained (121)[1I1] and $(\overline{112})[1 \overline{1} 1]$, as shown in Fig. 9. The $\{112\}<111\rangle$

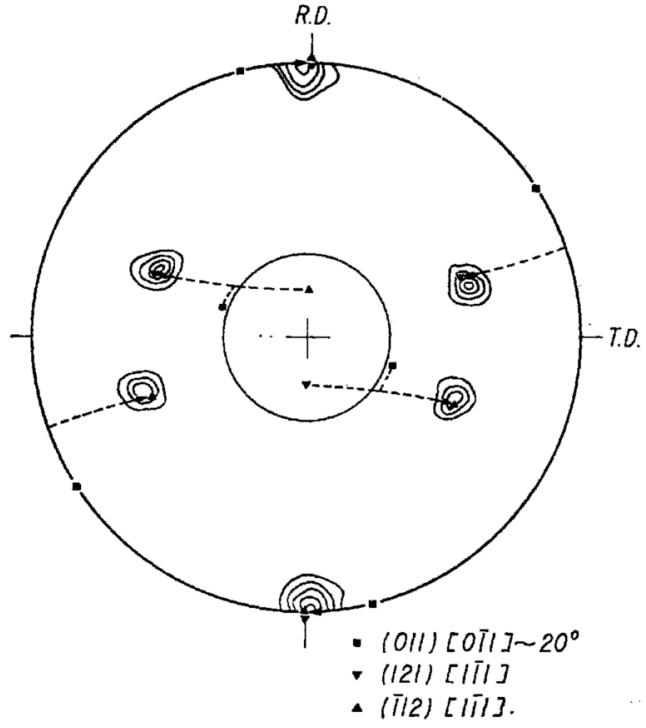

Fig. 9 Texture development in the (011) [12/2] single crystals rolled 70 pct. Separate components due to band formation attain a pair of $\{112\}<111>$ orientations.

orientation, which is one of the dominant orientations for metals of high stacking fault energy, was stable up to quite high reductions.

\section{Discussion}

In most of the theories concerning the development of rolling textures, the band formation has been ignored on the basis of the assumption that separate bands would behave like individual grains during deformation. In all the orientations adopted in this work, however, band formation causes clockwise and counterclockwise rotations about a common $\langle 111\rangle$ axis near the rolling or transverse direction. The above assumption seems to be inadequate, and the rotation by deformation banding should be taken into consideration in addition to the slip rotation.

Results obtained in this work give an important suggestion to the formation of the rolling-texture in polycrystalline aluminium. The orientations within the range between $\{111\}<110>$ and $\{112\}<110>$ tend to form deformation bands under plane strain deformation. If, by deformation banding, the crystals with these orientations are to rotate about the $\langle 111\rangle$ axis near the transverse direction as in the case of the (I11)[110] or (112)[110] orientation, the formation of a limited fiber texture ranging from $\{123\}<745>$ to $\{011\}<112\rangle$ would be realized. Figure 10 shows the distribution of orientation with banding tendency and the end fiber texture after the rotation. On the other hand, there are many other orientations with a banding tendency near the $\{011\}<111\rangle$ orientation. These are plotted on the octahedral pole figure of Fig. 11. If they rotate about the $\langle 111\rangle$ axis near the rolling direction as in the case of $(011)[1] 1]$ orientation, it can be expected that a limited fiber texture ranging from $\{112\}<111>$ to $\{123\}<745>$ is built up as shown in Fig. 11.

Both the limited fiber textures were proposed by 


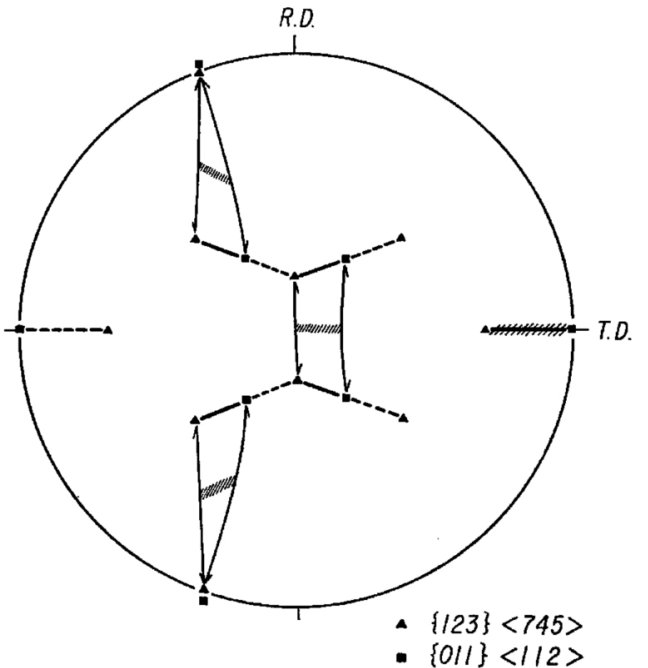

Fig. 10 Distribution of orientation with banding tendency resulting in the limited fiber texture ranging from $\{123\}<745>$ to $\{011\}<112>$.

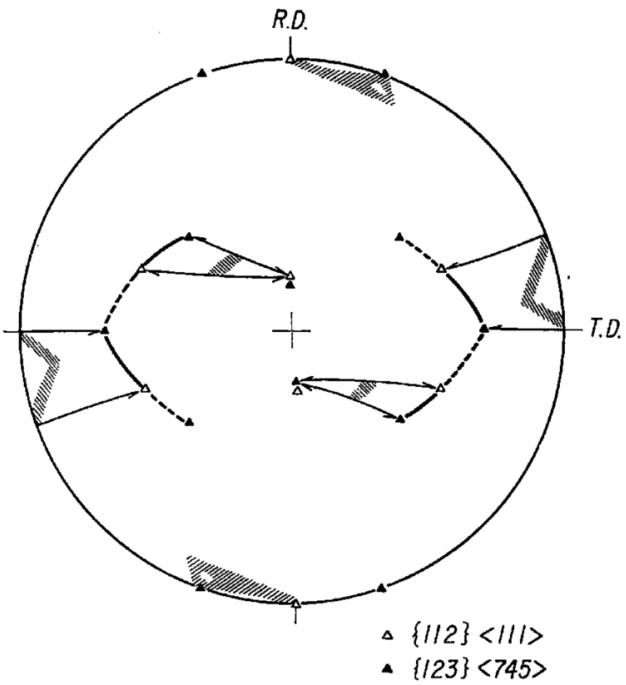

Fig. 11 Distribution of orientations with banding tendency resulting in the limited fiber texture ranging from $\{112\}<111>$ to $\{123\}<745>$.

Wassermann ${ }^{(18)}$ to interpret the copper-type pole figure, and the existence of orientations corresponding to these fiber textures was confirmed by means of electron diffraction $^{(16)(17)}$. Dillamore and Roberts ${ }^{(19)}$ considered that cross slip contributes to the development of the fiber textures in $f c c$ metals of high stacking fault energy. But our results indicate that the deformation banding is responsible for the formation of the fiber textures.

In polycrystalline aggregates, some of the grains continue to deform without occurrence of deformation bands until they attain the end orientations. Slip rotations during rolling have been analyzed by a number of investigators. Most of them have assumed that slip occurs in only one or two systems in the interior of a grain. This assumption ignores the compatibility of strains for

(18) J. Grewen und G. Wassermann : Acta Met., 3 (1955), 354.

(19) I. L. Dillamore and W. T. Roberts : Acta Met., 12 (1964), 281.

(20) G.I. Taylor : J. Inst. Metals, 62 (1938), 307.

(21) F. W. Bishop and R. Hill : Phil. Mag., 42 (1951), 414.

(22) F. W. Bishop and R. Hill : Phil. Mag., 42 (1951), 1298.

(23) R. O. Williams : Trans. Met. Soc. AIME, 242 (1968), 105. a given shape change. If it is assumed that the plastic flow of metals during rolling takes place in the condition of plane strain, it should be considered that, in addition to the stress, slip in individual grains must operate to coincide with the state of plane strain.

A $\{111\}<011>$ slip system in $f c c$ metals causes lattice rotation about $a<112\rangle$ axis. If a grain requires the operation of $n$ slip systems to deform in the condition of plane strain, it must rotate about an axis given by the following equation :

$$
\left\langle h k l>\infty \sum_{i=1}^{n}\left|d \gamma_{i}\right| \cdot<112>,\right.
$$

where $d \gamma_{i}$ is incremental shear in the $i$-th slip system. In a stable orientation $h, k$ and $l$ are all zero. Therefore, by repeating the process of determining $\langle h k l\rangle$ at a small degree of rotation or by following the orientational changes on the stereographic projection by plotting the calculated rotational directions, the end orientations under plane strain deformation are determined. Active slip systems necessary to ensure plane strain deformation can be estimated by Taylor ${ }^{(20)}$ or Bishop-Hill's ${ }^{(21)(22)}$ principle. And relative amounts of incremental shears for operative slip systems can also be calculated without difficulty, providing that the number of operative slip is six or less. If slip systems of more than six operate, relative amounts of slip shears cannot be determined. In such a case, the assumption that the slip shear is proportional to the resolved shear stress for each slip will not introduce large errors. In this analysis, Bishop-Hill's principle was applied to the estimation of active slip systems under plane strain deformation.

Results of this analysis indicate that most of the crystals rotate toward one of the orientations of $\{112\}<111>,\{123\}<745>$ and $\{011\}<112>$, and that a small amount of crystals rotates toward $\{011\}<100\rangle$. The former three orientations are dominant end orientations of the copper-type texture. $\{011\}<100>$ has been considered as one of the orientations corresponding to the saddle points ${ }^{(19)}$. The result of Williams ${ }^{(23)}$ indicates, however, that rolling textures of copper and aluminium have the $\{011\}$ $<100>$ component.

Both the experimental and theoretical analyses in this work show that $f c c$ metals tend to form the copper-type texture. Dillamore and Roberts ${ }^{(19)}$ claimed that in all $f c c$ metals the brass-type texture is developed initially by a normal mode of slip, and that the orientations deduced by rotation due to cross slip fit the copper-type texture. Contrary to the expectation of the cross-slip hypothesis, copper and aluminium crystals with the $\{011\}<112>$ orientations retain the initial orientation even after severe reductions ${ }^{(8)(24)(25)}$. Besides, existing evidence indicates that abundant cross slip is observed in brass ${ }^{(26)(27)}$. If the compatibility of strains for a given

(24) Y. C. Liu and W. R. Hibbard, Jr. : Trans. AIME, 197 (1953), 673.

(25) K. Ikeda and E. Tanaka : unpublished.

(26) R. Maddin, C. H. Mathewson and W. R. Hibbard, Jr. : Trans. AIME, 175 (1948), 86. 
shape change is taken into consideration, it is obvious that cross slip must be extensive depending not on stacking fault energy but on orientation. Haessner ${ }^{(28)}$ considered that cubic slip changes the $\{011\}<112\rangle$ orientation into $\{112\}<111\rangle$. However, there is no essential difference between Haessner and DillamoreRoberts' hypotheses, because an equal amount of primary and cross slip gives shear equivalent to cubic slip.

The present results, being imcompatible with the theories of Dillamore-Roberts and Haessner, are not con-

(27) R. Maddin, C. H. Mathewson and W. R. Hibbard, Jr. : Trans. AIME, 185 (1949), 527.

(28) F. Haessner : Z. Metallk., 54 (1963), 98.

(29) G. Wassermann : Z. Metallk., 54 (1963), 61. clusive in rejecting or supporting such theories as Wassermann's "twinning"(29) and Hu's "slip by partials"(2) theories. The present knowledge of the deformation mechanism resulting in the formation of rolling textures is quite unsatisfactory. It is desirable that, in future studies of the texture development, not only slip but also the other deformation modes should be practically examined.

\section{Acknowledgment.}

Experimental assistance from Y. Suzuki, M. Sugiyama and T. Shibuya is gratefully acknowledged. 